\title{
Uso de ferramentas de gestão na atividade leiteira: um estudo multicasos em Uberlândia, MG ${ }^{1}$
}

\author{
Marcos Aurélio Lopes ${ }^{2}$, Eduardo Mitke Brandão Reis ${ }^{3}$, Fabiana Alves Demeu ${ }^{4}$, Alan Andrade \\ Mesquita $^{5}$, Águida Garreth Ferraz Rocha ${ }^{6}$, Djalma Ferreira Pelegrini ${ }^{7}$, João Gabriel Knynichala Faria ${ }^{8}$, \\ Fernando Etiene Pinheiro Teixeira Junior ${ }^{9}$
}

\begin{abstract}
Resumo: Objetivou-se analisar a aplicabilidade das ferramentas de gestão matriz GUT (gravidade, urgência e tendência), Brainstorming, PDCA (plan, do, check, action), diagrama de Ishikawa e 5W2H, visando o levantamento e a correção de pontos falhos a partir do diagnóstico de propriedades produtoras de leite. Os dados utilizados foram provenientes de quatro propriedades, localizadas na região de Uberlândia, MG, de agosto a dezembro de 2014, sendo coletados a partir de um formulário semiestruturado, contendo 549 questões. As respostas foram obtidas por meio da observação, bem como por entrevistas com os proprietários. Utilizando-se a matriz GUT, os principais pontos fracos encontrados, em ordem decrescente, foram: não realização de exames de brucelose e tuberculose, ausência de pastejo rotacionado, falta de volumoso para a época seca, excesso de proteína na ração concentrada e presença de lama nas áreas de descanso. Os principais pontos fortes encontrados foram: boa qualidade do leite, manejo adequado de vacas secas, cuidados com recém-nascidos, uso de tecnologias (inseminação artificial, ordenhadeira mecânica, cerca elétrica) e realização de controle zootécnico. A partir do ranqueamento dos pontos fracos, e utilizando a ferramenta de gestão Brainstorming, definiu quais ferramentas de gestão seriam utilizadas: PDCA, 5W2H e/ou Diagrama de Ishikawa, visando corrigi-los ou, pelo menos, amenizá-los. Concluiu-se que é possível adequar as ferramentas de gestão à pecuária leiteira, podendo ser usadas de forma contínua para o gerenciamento ou de forma pontual no estabelecimento de um plano ou projeto. Recomenda-se que elas sejam utilizadas, pois pontos fracos poderão ser identificados e corrigidas as ineficiências, contribuindo no aumento da rentabilidade e da lucratividade. Entretanto, são necessários conhecimentos de gestão e zootécnicos para aplicação de tais ferramentas em fazendas leiteiras.
\end{abstract}

Palavras Chave: Diagnóstico organizacional; Gerenciamento; Pecuária de leite; Qualidade.

\section{Using management tools in dairy farm: a multicase study in Uberlândia, MG}

\begin{abstract}
The scope of this research was to accomplish the diagnosis of dairy cattle properties and analyze the applicability of several management tools, GUT array (severity, urgency and tendency), brainstorming, Ishikawa Diagram, PDCA cycle (plan, do, check, action), 5W2H, aimed at lifting and and correct weaknesses. Sought, identify the strengths. The data used were from four properties, located in the region of Uberlândia, MG, from August to December 2014, in which data and information were collected using a semi-structured form, containing 549 issues, through observation, as well as by responses obtained with the owners during the diagnosis. Using the array GUT, were made a ranking the main weaknesses found, in descending order: no tests of brucellosis and tuberculosis, the absence of rotational grazing, lack of roughage to the dry season, excess protein in the diet and the presence of mud in the rest areas. The main strengths found were: good quality of milk, proper management of dry cows, newborn care, use of technologies (artificial insemination, mechanical milkmaid, electric fence) and realization of zootechnical bookkeeping. From the seeding of the weak points, and using the Brainstorming management tool, defined which management tools would be used: PDCA, 5W2H and/or Ishikawa diagram, aiming to correct them or at least ease them. It was concluded that it is possible to adapt the management tools to dairy livestock, and can be used continuously for management or on a punctual basis in the establishment of a plan or project. It is recommended that they be used because weak points can be identified and corrected inefficiencies, contributing to the increase in profitability and profit. However, knowledge of management and zootechnical is necessary for the application of such tools in dairy farms.
\end{abstract}

Key words: Dairy cattle; Management; Milk quality; Organizational diagnosis.

\footnotetext{
${ }^{1}$ Submetido em 17/05/2017 e aprovado em 09/02/2018

${ }^{2}$ Dout or emZootecnia; Professor Titular, Universidade Federal de Lavras (UFLA), Departamento de Medicina Veterinária, Lavras-MG, CEP: 37200-000; E-mail: malopes@dmv.ufla.br; bolsista do CNPq

${ }^{3}$ Dout or em Ciências Veterinárias; Professor, Universidade Federal do Acre(UFAC), Centro de Ciências Biológicas e da Natureza, Rio BrancoAC, CEP: 69.900-000; E-mail: edumitke@gmail.com

${ }^{4}$ Mestre em Ciências Veterinárias; Professor, Instituto Federal de Rondônia (IFRO), Departamento de Zootecnia, Ariquemes-RO, CEP: 76.878899; E-mail: fabiana.alves@ifro.edu.br

${ }^{5}$ Doutor em Ciências Veterinárias; Zootecnista, Universidade Federal de Lavras (UFLA), Departamento de Zootecnia, Lavras-MG, CEP: 37.200-000; E-mail: alan.mesquita@dzo.ufla.br

${ }^{6}$ Doutor em Engenharia de Produção; Professor, Universidade Federal de Uberlândia (UFU), Departamento de Medicina Veterinária e Zootecnia, Uberlândia-MG, CEP: 38.405-314; E-mail: aguidagarreth @gmail.com

${ }^{7}$ Doutor em Geografia; Pesquisador, Empresa de Pesquisa de Minas Gerais (EPAMIG), Belo Horizonte-MG, CEP: 31.170-495; E-mail: djalmapelegrini@uol.com.br

${ }^{8}$ Médico Veterinário; Aut ônomo, Uberlândia-MG, CEP: 38.405-314; E-mail: jgknychala @hotmail.com

${ }^{9}$ Mestre em Zootecnia; Professor, Universidade Estadual de Montes Claros (UNIMONTES), Departamento de Ciências da Administração,

Montes Claros-MG, CEP: 39.401-089; E-mail: fernandoetiene82@ hotmail.com
} 


\section{Introdução}

Apesar de o Brasil ser o quarto maior produtor de leite bovino do mundo, a produtividade anual ao redor de $1.680 \mathrm{~L} /$ vaca é baixa quando comparada à dos Estados Unidos, de 9.850 L/vaca, da União Europeia de $6.140 \mathrm{~L} / v a c a$, da Argentina de $5.830 \mathrm{~L} / \mathrm{vaca}$ e da Nova Zelândia de $4060 \mathrm{~L} / \mathrm{vaca}$ (Pereira et al., 2016).

A atividade leiteira brasileira tem índices zootécnicos, econômicos e de produtividade muito baixos, tornando a atividade pouco atrativa. Some-se a isso o despreparo do setor produtivo, principalmente na área de gestão (Novo e Schiffler, 2006). A falta de preparo do produtor o afeta negativamente, proporcionando baixo retorno econômico, e desmotivando-o na continuação da produção de leite, havendo a necessidade dele se adaptar, pensar e agir como um empresário rural, com visão gerencial de sua atividade (Crepaldi, 1998), independente de ser pequeno, médio ou grande produtor (Lopes et al, 2004).

O planejamento da atividade leiteira é um instrumento essencial na busca da eficiência e indispensável para alcançar o desenvolvimento sustentável (Silva et al., 2015). As ferramentas de gestão podem ser utilizadas dentro das propriedades rurais como estratégia de aumento da produtividade (Parré et al., 2011). Não parece haver dúvidas de que elas afetarão, de forma profunda, a viabilidade e sustentabilidade da agricultura familiar (Batalha et al. 2005). Acredita-se que a sua aplicação possa melhorar a compreensão da atividade como um todo, melhorando, assim, a administração e o manejo da propriedade rural, tendo como consequência o aumento de lucratividade e da rentabilidade.

Uma boa gestão faz com que os produtores rurais tenham condições de desenvolver o setor agropecuário, e as ferramentas de gestão podem auxiliar nesta tarefa, contribuindo com melhorias em suas condições socioeconômicas, decisões estratégicas e avanços na atividade produtiva (Farias et al., 2013).

As ferramentas de gestão são técnicas para uso empresarial visando, entre outros, solução de problemas gerenciais, e seu uso pode auxiliar empresários na tomada de decisões dentro de seu processo produtivo, pois os orientam na observação, identificação e análise de problemas (Meireles, 2001) e quando aplicadas geram possibilidades de obter maior lucro em sua atividade fim (Roberti e Santana, 2013), podendo, também, ser utilizadas na pecuária de leite.

Bonamigo et al. (2014), Duarte et al. (2013), Santana e Araújo (2011) e Sousa et al. (2011), pesquisaram sobre o uso de ferramentas de gestão no agronegócio; porém, nenhum dos estudos se refere à avaliação e uso de tais ferramentas na bovinocultura leiteira. Raros são os que as utilizam no setor primário (Alves et al., 2007).

Diante da importância e da atual conjuntura em que se encontra a pecuária leiteira no Brasil e a escassez de artigos científicos sobre o do tema, objetivou-se analisar a aplicabilidade das ferramentas de gestão matriz GUT (gravidade, urgência e tendência), Brainstorming, PDCA (plan, do, check, action), diagrama de Ishikawa e $5 \mathrm{~W} 2 \mathrm{H}$, visando o levantamento e a correção de pontos falhos a partir do diagnóstico de propriedades produtoras de leite.

\section{Material e Métodos}

A pesquisa foi realizada em quatro sistemas de produção de leite localizados no município de Uberlândia, Minas Gerais, de agosto a dezembro de 2014, por uma equipe multidisciplinar composta por médicos veterinários, zootecnistas e pesquisadores. Para definir as propriedades foi levado em consideração o acesso da propriedade, receptividade do gerente, disposição em acatar o que seria proposto e conhecimento sobre o seu sistema produtivo.

Para realização do diagnóstico, utilizou-se um formulário semiestruturado (Lopes et al., 2016), desenvolvido especificamente para esse fim com o intuito de levantar os pontos fortes e fracos dos sistemas estudados, composto por 549 questões divididas em tópicos: caracterização do produtor e da propriedade (52 questões), caracterização do rebanho (12) e caracterização da produção de leite (485). Nesse último tópico inclui-se o sistema de produção, práticas agrícolas, manejo nutricional, infraestrutura, escrituração zootécnica, identificação dos animais, manejo reprodutivo, qualidade do leite, manejo de ordenha, criação de bezerras, controle sanitário e manejo ambiental. Tal formulário possui, ainda, um check-list de fotos, o qual auxiliará os pesquisadores na expectativa de registrar detalhes difíceis de serem descritos e lembrados, e que serão úteis no levantamento dos pontos fortes e fracos. 
Em um segundo momento, houve o levantamento dos pontos fortes e fracos encontrados nos sistemas de produção estudados. Para tal, foi utilizada a ferramenta de gestão brainstorming (Andrade e Boff, 2014). Nesta etapa, cada membro da equipe multidisciplinar, expôs suas opiniões em relação a cada item analisado no formulário de diagnóstico.

Após definir os pontos fracos, por meio da ferramenta de gestão brainstorming (Andrade e Boff, 2014), cada pesquisador, individualmente, avaliou e pontuou de zero a cinco cada item que considerou mais grave, mais urgente e com maior tendência a piorar, utilizando a ferramenta de gestão matriz GUT (Meireles, 2001).

Definidos os pontos fracos, por meio da ferramenta de gestão Brainstorming (Andrade e Boff, 2014), cada pesquisador, individualmente, avaliou e pontuou de zero a cinco cada item que considerou mais grave, mais urgente e com maior tendência a piorar, utilizando a ferramenta de gestão Matriz GUT (Meireles, 2001). Os itens foram listados em uma planilha, com as respectivas notas, e estimada uma média para as diferentes notas atribuídas por cada pesquisador. Em seguida, os pontos fracos, a partir da pontuação média, foram ranqueados em ordem decrescente.

Visando a indicação da ferramenta de gestão mais adequada para a solução daqueles problemas, a equipe fez uso, novamente, da ferramenta brainstorming (Coletti et al., 2010) e selecionou o Diagrama de Ishikawa (Ishikawa, 1985), 5W2H (Polacinski et al, 2012) e PDCA (Aguiar, 2006) na busca de corrigir ou, ao menos, minimizar os pontos fracos diagnosticados, e consequentemente, o aumento da rentabilidade.

\section{Resultados e Discussão}

Os pontos fracos encontrados durante a realização do diagnóstico, listados em ordem descrente, pela média da pontuação atribuída após a utilização da matriz GUT (Meireles, 2001), podem ser observados na Tabela 1. As maiores pontuações indicam os pontos fracos que deverão ser priorizados na tentativa de equacioná-los ou minimizá-los, pois possuem maior gravidade, maior urgência em ser resolvidos e maiores tendências de se agravarem. Em alguns deles, observa-se altos valores de desvio padrão, o que denota a divergência de opiniões entre os pesquisadores. Os principais foram: não realização de exames de brucelose e tuberculose, ausência de pastejo rotacionado, falta de volumoso para a época seca, excesso de proteína na ração concentrada e presença de lama nas áreas de descanso.

A realização do diagnóstico foi fundamental para que se pudessemidentificar os pontos fracos, que podem comprometer o sucesso produtivo e econômico dos sistemas de produção estudados. Isso é benéfico, pois, para solucioná-los, não há necessidade de grandes investimentos ou esforços demasiados, fato esse que torna promissor os sistemas de produção. Com base nos pontos elencados pode-se realizar um plano de ação e metas a serem cumpridas no curto, médio e longos prazos. A coleta de dados, por meio do diagnóstico realizado, visou agilizar as tomadas de decisões dentro do sistema de produção de leite.

O uso das ferramentas de gestão se torna útil dentro da propriedade, atuando na identificação do problema, e ordenando-os conforme sua gravidade, urgência e tendência a piorar, para que possam ser solucionados. Portanto, a aplicação desses instrumentos poderá abrir caminhos para construção de um plano estratégico de gestão, a partir de metodologias de diagnóstico organizacional e sistema de administração,além de contribuir para a capacitação de recursos humanos em administração de empreendimentos rurais. Wolf (2012) relata que a não utilização de ferramentas para auxílio na gestão tem diversas razões, entre elas o custo, a falta de gerenciamento de tempo e a falta de compreensão do assunto.

\subsection{Primeiro ponto fraco: falta de controle nas enfermidades brucelose e tube rculose bovina}

Em $75 \%$ dos sistemas de produção estudados não foi possível observar métodos de controle sobre doenças infecciosas. A brucelose e a tuberculose são importantes zoonoses de distribuição mundial com grande relevância na bovinocultura (principalmente leiteira) que acarretam prejuízos econômicos e problemas na saúde pública (Mocci et al., 2014). A importância econômica atribuída a essas doenças está baseada nas perdas diretas resultantes da morte de animais, da queda no ganho de peso, diminuição da produção de leite, do descarte precoce e condenação de carcaças no abate (Souza et al., 2013). 
Tabela 1 Pontuação atribuída por meio da matriz GUT a cada ponto fraco identificado nos sistemas de produção de leite estudados em Uberlândia, de agos to a dezembro de 2014

\begin{tabular}{|c|c|c|c|c|c|c|c|c|c|}
\hline \multirow{2}{*}{ Pontos fracos } & \multicolumn{8}{|c|}{ Nota atribuída pe los pesquisadores* } & \multirow{2}{*}{$\begin{array}{l}\text { Desvio } \\
\text { padrão }\end{array}$} \\
\hline & 1 & 2 & 3 & 4 & 5 & 6 & 7 & $\overline{\text { Média }}$ & \\
\hline Falta de controle de nas enfermidades brucelose e tuberculose bovinas & 125 & 60 & 125 & 27 & 125 & 18 & 36 & 89,00 & 49,64 \\
\hline Ausência de past ejo rotacionado & 100 & 36 & 125 & 48 & 125 & 100 & 36 & 85,00 & 40,27 \\
\hline Falta de volumoso para a época seca & 125 & 18 & 100 & 100 & 50 & 80 & 80 & 81,86 & 35,50 \\
\hline Excesso de proteína na ração comercial & 125 & 36 & 100 & 100 & 4 & 125 & 64 & 75,57 & 46,21 \\
\hline Presença de lama nas áreas de descanso & 125 & 45 & 80 & 48 & 12 & 80 & 125 & 73,57 & 42,10 \\
\hline Não faz vacinação de clostridiose, paratifo e leptospirose & 80 & 36 & 64 & 48 & 125 & 64 & 64 & 68,71 & 28,46 \\
\hline Ausência de calagem e adubação & 125 & 36 & 64 & 24 & 125 & 8 & 36 & 67,71 & 47,64 \\
\hline Baixa proporção de vacas em lactação & 80 & 100 & 64 & 15 & 45 & 80 & 36 & 57,71 & 29,56 \\
\hline Pastagem de baix a qualidade & 100 & 12 & 80 & 27 & 64 & 27 & 36 & 57,00 & 32,38 \\
\hline Uso incorreto de sêmen sexado & 25 & 125 & 8 & 100 & 16 & 125 & 100 & 54,57 & 52,64 \\
\hline Pastejo diferido & 8 & 36 & 64 & 80 & 50 & 64 & 36 & 48,29 & 23,90 \\
\hline Troca local de casinha tropical para bezerras somente quando forma barro & 27 & 36 & 45 & 45 & 1 & 100 & 125 & 46,29 & 43,17 \\
\hline Compra de ração comercial & 25 & 36 & 100 & 16 & 4 & 48 & 36 & 45,29 & 30,99 \\
\hline Não vermifuga novilhas e vacas no pré-parto & 60 & 24 & 48 & 64 & 18 & 48 & 48 & 44,29 & 17,22 \\
\hline Não faz análises de água & 4 & 64 & 36 & 64 & 2 & 80 & 100 & 43,71 & 37,43 \\
\hline Critérios de agrupamentos do rebanho & 27 & 18 & 27 & 125 & 9 & 27 & 48 & 40,14 & 39,24 \\
\hline Não tem acasalamento genético & 80 & 48 & 12 & 24 & 8 & 32 & 64 & 35,43 & 26,92 \\
\hline Baixa lotação de pastagem & 48 & 18 & 32 & 36 & 64 & 36 & 12 & 34,57 & 17,51 \\
\hline Joga fora o leite de transição & 25 & 80 & 48 & 4 & 25 & 36 & 12 & 34,57 & 25,34 \\
\hline Não possui recomendação veterinária para tratamento da mastite & 9 & 64 & 1 & 48 & 8 & 60 & 100 & 33,00 & 36,81 \\
\hline Local inadequado para casinha das bezerras & 48 & 18 & 12 & 12 & 1 & 100 & 125 & 32,57 & 48,78 \\
\hline Critério errôneo para controle de infestação de ectoparasitas & 48 & 24 & 12 & 18 & 12 & 48 & 100 & 32,29 & 31,60 \\
\hline Descorna realizada tardiamente & 100 & 16 & 8 & 24 & 1 & 9 & 64 & 31,57 & 36,59 \\
\hline Não utiliza dieta de transição & 36 & 24 & 36 & 12 & 9 & 27 & 64 & 31,00 & 18,43 \\
\hline Presença de plantas invasoras nos pastos & 48 & 24 & 48 & 18 & 12 & 18 & 18 & 30,86 & 15,04 \\
\hline Posição inadequada de bezerreiros & 48 & 4 & 18 & 27 & 2 & 9 & 80 & 28,14 & 28,31 \\
\hline Falta de manutenção de cercas & 64 & 8 & 12 & 18 & 16 & 12 & 60 & 27,14 & 24,05 \\
\hline Não faz vacinação pré parto & 27 & 32 & 27 & 1 & 12 & 18 & 64 & 27,14 & 19,86 \\
\hline Árvores com copa densa na pastagem & 36 & 12 & 12 & 48 & 12 & 27 & 48 & 25,71 & 16,50 \\
\hline Fornecimento incorreto de concentrado para bezerras & 25 & 24 & 8 & 18 & 2 & 48 & 64 & 21,29 & 21,95 \\
\hline Produção de leite vaca/dia & 48 & 36 & 18 & 12 & 1 & 64 & 12 & 20,71 & 22,72 \\
\hline Identificação tardia de bezerras & 48 & 16 & 12 & 12 & 1 & 36 & 36 & 19,57 & 17,02 \\
\hline Não possui constânciana pesagem das bezerras & 27 & 16 & 18 & 18 & 2 & 36 & 36 & 19,29 & 12,14 \\
\hline Não realiza biocarrapatograma & 4 & 64 & 18 & 8 & 20 & 18 & 8 & 20,00 & 20,36 \\
\hline Não faz práticas de conservação de solo & 27 & 16 & 8 & 18 & 1 & 12 & 48 & 18,00 & 15,32 \\
\hline Intervalo do controle leit eiro & 27 & 4 & 8 & 27 & 1 & 6 & 64 & 19,86 & 22,32 \\
\hline Não realiza OPG(ovos por gramas) & 12 & 36 & 1 & 36 & 1 & 12 & 12 & 14,14 & 14,71 \\
\hline Cochos de sal descobertos & 27 & 16 & 1 & 18 & 1 & 27 & 12 & 10,86 & 10,78 \\
\hline Não possui banco de colostro & 12 & 48 & 1 & 12 & 3 & 2 & 27 & 14,86 & 17,13 \\
\hline Distância atéo laticínio & 1 & 4 & 36 & 12 & 1 & 2 & 1 & 13,00 & 12,90 \\
\hline Não avalia a qualidade do colostro & 12 & 24 & 9 & 4 & 2 & 3 & 18 & 11,14 & 8,30 \\
\hline Intervalo entre ordenhas & 12 & 18 & 4 & 2 & 1 & 18 & 8 & 7,00 & 7,19 \\
\hline Ausência de irrigação & 18 & 2 & 4 & 9 & 1 & 2 & 4 & 6,00 & 6,02 \\
\hline Armazenamento do leite por $48 \mathrm{~h}$ & 9 & 2 & 1 & 2 & 1 & 1 & 2 & 2,86 & 2,91 \\
\hline
\end{tabular}

*Multiplicação das notas de zero a cinco para os quesitos gravidade $(\mathrm{G})$, urgência $(\mathrm{U})$ e tendência $(\mathrm{T})$

A brucelose é uma doença crônica de animais domésticos, selvagens e seres humanos causada pela Brucella ssp, que são bactérias intracelulares com grande capacidade para invadir, sobreviver por longos períodos de tempo e se multiplicarem dentro de células hospedeiras (Poester et al., 2013). Em rebanhos infectados por brucelose, estima-se uma diminuição da produção de carne e leite na ordem de $25 \%$ (Miranda et al., 2008) e um decréscimo da produção de bezerros, em média, de 15\% (Bernués et al., 1997). Segundo Santos et al. (2013), a cada 1\% de variação na taxa de prevalência de brucelose bovina no Brasil, estima-se em R $\$ 155$ milhões o custo da doença. Essas perdas econômicas comprometem mais de
$0,3 \%$ do produto interno bruto (PIB) brasileiro gerado por animais de produção

A tuberculose é causada pelo Mycobacterium bovis que é um patógeno de importância significativa na pecuária. Sabe-se também que pode causar a doença em seres humanos, o que tem levantado preocupações em relação à ocorrência de zoonoses em humanos, especialmente aqueles que vivem na interface homem-animal (Michel et al., 2010)

As propriedades pesquisadas nunca tiveram um planejamento de combate à brucelose e a tuberculose bovina, sendo fundamental estimar a prevalência de ambas as enfermidades, através de 
exames de diagnóstico, para que seja possível identificar os animais contaminados, e fornecer subsídios para a melhor implementação e gestão do Programa Nacional de Controle e Erradicação de Brucelose e Tuberculose (PNCEBT, 2006), pois os prejuízos causados por elas são enormes.

\subsection{Ferramenta proposta para solucionar o primeiro ponto fraco: ciclo PDCA}

A ferramenta de gestão proposta para a minimização do ponto fraco "Falta de controle nas enfermidades brucelose e tuberculose bovina" foi o Ciclo PDCA, pois o problema requer acompanhamento contínuo. De acordo com a Instrução Normativa - IN no 10 , de 3/3/2017 do PNECBT (BRASIL, 2017), as ações consistem na vacinação de bezerras entre os três e oito meses de idade contra a brucelose, controle do trânsito de animais e a certificação de propriedades livres de brucelose e/ou de tuberculose. Acredita-se eu para se atingir tais metas é necessário o um acompanhamento contínuo. Estabeleceu-se que, uma vez implantada, haverá necessidade de controle individual dos animais através de fichas e planilhas; que estarão sempre em uso, obedecendo a um ciclo de melhoria contínua. Segundo Aguiar (2006), o uso desta ferramenta passa por quatro etapas, com início na identificação do problema e finalizando com a avaliação. O ciclo PDCA é dividido em: Plan (Planejamento) que consiste no estabelecimento da meta ou objetivo a ser alcançado; Do (Execução) é o trabalho de realização da meta e do plano pré-definidos; Check (Verificação da execução do trabalho) durante e após a execução; Action (Ação) transformar o plano que deu certo na nova forma de realização das atividades dentro da empresa. Deve ser utilizado em problemas frequentes, que necessitem de monitoração constante e que seja mais complexa (Antunes e Essels, 1999).

\subsection{Segundo ponto fraco: ausência de pas tejo rotacionado}

Em 75\% dos sistemas de produção pesquisados observou-se que não havia pastejo rotacionado, o que estava acarretando problemas, como por exemplo, a degradação de pastagens. $\mathrm{O}$ pastejo em sistema rotacionado nada mais é do que dividir a área em piquetes de acordo com a forrageira utilizada, levando-se em consideração o número de dias de descanso e os dias de ocupação da pastagem (tempo que os animais permanecem no piquete).

Nascimento e Carvalho (2011) considera que a principal causa da degradação das pastagens é advinda do manejo animal inadequado e à carência de nutrientes no solo, processo evolutivo de perda de vigor das forragens $\mathrm{e}$ da produtividade, e capacidade de recuperação natural das pastagens, tornando-a incapaz de sustentar os níveis de produção e qualidade exigidos pelos animais, bem como superar os efeitos nocivos de pragas, doenças e plantas invasoras. Com isso, a degradação da pastagem faz com que haja redução na produtividade bovina e aumento do custo de produção (Macedo, 2009). Há diversas técnicas para recuperação de pastagens degradadas, entre elas o pastejo rotacionado, que contribui na oferta de matéria verde para os animais, de forma mais sustentável e com menos impactos ao meio ambiente (Peron e Evangelista, 2004).

É uma tendência do produtor rural brasileiro o alto investimento em genética e o baixo investimento em formação de pastagens, sendo que esta combinação acarreta uma das principais características da bovinocultura brasileira: a alta produção decorrente da grande quantidade de animais, porém associada à baixa produtividade, decorrente do fornecimento insuficiente de substrato energético e proteicos, que por sua vez é devido a uma pastagem de má qualidade e à ausência de planejamento e manejo nutricional (Ribeiro et al., 2016).

Resultados de pesquisas evidenciam os bons rendimentos dos sistemas de produção que adotam o pastejo rotacionado (Abdalla et al., 1999; Houtert e Sykes, 1999).

\subsection{Ferramenta proposta para solucionar o segundo ponto fraco: $5 \mathrm{~W} 2 \mathrm{H}$}

Para a solução do ponto fraco "ausência de pastejo rotacionado", há a necessidade de se tomar a decisão de adotar tal tecnologia e, para tanto, a ferramenta de gestão proposta para foi a 5W2H (Lisboa e Godoy, 2012), pois esse tipo de decisão não necessita do envolvimento de grande quantidade de pessoas, nem possui complexidade para que possa ser tomada a decisão. Segundo Cheng e Meli Filho (2007), essa ferramenta foi criada por profissionais da indústria automobilística do Japão para auxiliar na 
utilização do PDCA, principalmente na fase de planejamento.

A ferramenta $5 \mathrm{~W} 2 \mathrm{H}$ atua como referência para sustentar as decisões. Dessa forma, permite a realização do acompanhamento, do incremento ou desenvolvimento de um determinado projeto (Oliveira, 1996). Um exemplo de aplicação da ferramenta para a proposta de solução do problema de bezerreiro coletivo no sistema de produção estudado é apresentado a seguir.

a) What (o que?): Decidir sobre implantar um sistema de pastejo rotacionado.

b) When (quando?): Imediatamente.

c) Who (quem?): Técnico do sistema de produção de leite.

d) Where (onde?): Avaliação do local após análise de solo e proximidade do curral.

e) Why (por quê?): Melhorar o sistema de pastejo e da qualidade das forrageiras.

f) How (Como?) Modificar o sistema de pastejo extensivo para pastejo rotacionado através do uso de piquetes e cercas elétricas.

g) How Much (quanto custa?): Avaliar de acordo com a quantidade de animais que participarão do sistema rotacionado e da análise do solo.

\subsection{Terceiro ponto fraco: falta de volumoso para a época seca}

Em 100\% dos sistemas de produção estudados foi observado que não havia quantidade de volumoso suficiente para a alimentação do rebanho durante a época seca, pois já é sabido que, a partir do momento em que se inicia a época seca do ano, há um declínio da disponibilidade de forragem.

O período chuvoso da região central do país, onde encontra-se o triangulo mineiro, é caracterizado pela ocorrência de temperaturas e índices pluviométricos elevados, com altas taxas de evapotranspiração. $\mathrm{Na}$ época seca, onde o fotoperíodo é mais curto, com baixas temperaturas noturnas e a baixa umidade, devido à menor pluviosidade, o crescimento das gramíneas pode ser limitado, promovendo, assim, comportamento estacional das forrageiras (Valle et al, 2000). A estacionalidade é caracterizada por variações na disponibilidade e qualidade da forragem em resposta às alterações nas condições climáticas, as quais não permitem que as plantas forrageiras tenham crescimento uniforme durante o ano (Reis e Rosa, 2001).
Diante do exposto, e de acordo com Oliveira et al. (2016), percebe-se que há necessidade de se ter um planejamento para a época seca, pois a disponibilidade de matéria seca (MS) por área serve para definir a taxa de lotação da pastagem. Para evitar esse ponto fraco é importante se buscar alternativas de volumosos na época da estação seca, que sejam economicamente viáveis, e muitos produtores tem utilizado a cana-deaçúcar (Saccharum officinarum L.) na alimentação de ruminantes. Ela, segundo Moreira (1983), tem sido disseminada por todo território nacional pelo fácil cultivo e grande produção de massa verde, facilitando a utilização na alimentação de bovinos Devido ao alto potencial de produção, principalmente na época seca do ano, merece atenção especial, e suas limitações nutricionais devem ser consideradas e corrigidas, permitindo que os animais apresentem desempenho similar ao obtido com outros volumosos, e com menor custo.

Em virtude do baixo conteúdo de proteína e alta concentração de carboidratos solúveis, Abreu et al. (2015) recomendaram suplementação da cana-de-açúcar com nitrogênio não protéico. $\mathrm{O}$ fornecimento de ureia visa atender diretamente à necessidade da microflora ruminal por nitrogênio (Borges et al., 2016).

Souza et al. (2015) avaliaram o efeito da adição de teores crescentes de ureia $(0,0 ; 0,5$ e $1,0 \%$ ) na matéria natural da cana-de-açúcar sobre a produção e composição do leite e eficiência alimentar. Os autores não observaram efeito da suplementação dos teores de ureia sobre a produção e composição do leite, mas a eficiência alimentar foi melhor nas dietas em que se utilizou ureia.

Santiago et al. (2013) avaliaram o efeito da adição de $0,4,8$ e $12 \mathrm{~g} \mathrm{~kg}^{-1}$ de cana de uma mistura de ureia e sulfato de amônio (9:1) Os autores concluíram que, em dietas com cana-deaçúcar para vacas leiteiras com produção abaixo de $15 \mathrm{~kg}$ leite por dia, as concentrações de ureia podem ser aumentadas de 0 a $12 \mathrm{~g} \mathrm{~kg}^{-1}$ de matéria natural sem prejudicar o desempenho, com diminuição da ingesta, melhorando, assim, seu desempenho.

\subsection{Ferramenta proposta para solucionar o terceiro ponto fraco: $5 \mathrm{~W} 2 \mathrm{H}$}

Para a solução do ponto fraco "Falta de volumoso para a época seca" há a necessidade de 
se tomar a decisão de adotar a implantação de um canavial. e, para tanto, a ferramenta de gestão proposta para foi a 5W2H (Lisboa e Godoy, 2012), pois esse tipo de decisão não necessita do envolvimento de grande quantidade de pessoas, nem possui grande complexidade para ser solucionada. Da mesma forma que o ponto fraco anterior, trata-se de uma atividade que não envolve muitas pessoas e nem complexidade em sua execução. Assim, é necessário, responder cada pergunta abaixo, para que sua utilização tenha sucesso.

a) What (o que?): Decidir sobre implantar um canavial.

b) When (quando?): Imediatamente.

c) Who (quem?): Responsável pelo sistema de produção.

c) Where (onde?): Nas áreas onde não há pastagens utilizadas paras os sistemas de produção.

d) Why (por quê?): Evitar falta de volumoso para o rebanho na época seca do ano.

e) How (Como?): Plantando mudas através de maquinário ou carro de boi.

f) How Much (quanto custa?): As mudas podem ser conseguidas na própria região. Haverá gasto com preparo da terra.

\subsection{Quarto ponto fraco: excesso de proteína fornecido aos animais}

Em 100\% dos sistemas de produção estudados percebeu-se que havia excesso de proteína fornecido aos animais, ou seja, os animais estavam consumindo mais proteína do que necessitavam. Em estudo realizado por Gomide et al. (2001), os pesquisadores obtiveram produção de leite média de $11,0 \mathrm{~kg}$ por vaca por dia, corrigida a $4 \%$ de gordura, com exigências diárias de PB, NDT, FDN, Ca e P, em kg por vaca, segundo NRC (1988), respectivamente de 1,39; 7,$3 ; 6,0 ; 0,048$ e $0,035.14,3,12,4$

O nível de produtividade de bovinos depende, entre outros fatores, do potencial genético do animal e do consumo de nutrientes; consumo este, que pode ser limitado pelo alimento, pelo animal ou pelas condições de alimentação (Cavalcante et al.., 2005). Segundo Mertens (1994), em torno de 60 a 90\% das variações no desempenho animal podem ser atribuídas às alterações no consumo de nutrientes, e de 10 a $40 \%$, às mudanças na digestibilidade dos alimentos.

A proteína é o segundo nutriente mais exigido pelos ruminantes. As exigências proteicas dos ruminantes são atendidas mediante a absorção intestinal de aminoácidos provenientes, principalmente, da proteína microbiana sintetizada no rúmen e da proteína dietética nãodegradada no rúmen (Valadares Filho e Valadares, 2001). Tanto a deficiência, como o excesso, de proteína na dieta pode reduzir o consumo; a deficiência, pelo não atendimento aos requerimentos dos microrganismos ruminais e o excesso, pela toxidez pela liberação de amônia, que aumenta o teor de uréia, via urina, constituindo em desperdício de proteína (Cavalcante et al., 2005).

Os elementos ricos em proteínas têm, em geral, preços mais elevados do que os alimentos ricos em carboidratos e pobres em proteínas. Por esse motivo, procura-se conhecer o mínimo que se deve fornecer aos animais. Além desses fatos, o fornecimento inadequado de proteínas pode levar a um desempenho inferior dos animais.

\subsection{Ferramenta proposta para solucionar o quarto ponto fraco: $5 \mathrm{~W} 2 \mathrm{H}$}

Para solucionar o ponto fraco "Excesso de proteína fornecida aos animais" foi proposta a ferramenta de gestão 5W2H. Da mesma forma que o ponto fraco anterior, trata-se de uma atividade que não envolve muitas pessoas e nem complexidade em sua execução. Assim, é necessário, responder cada pergunta abaixo, para que sua utilização tenha sucesso.

a) What (o que?): Reformular a dieta fornecida aos animais

b) When (quando?): Imediatamente.

c) Who (quem?): Técnico responsável pelo sistema de produção.

c) Where (onde?): No setor de compras.

d) Why (por quê?): Evitar redução de consumo e perdas econômicas por gastos excessivos com um componente caro da alimentação animal como a proteína.

e) How (Como?): Fabricação da própria ração.

f) How Much (quanto custa?): Menos do que a aquisição da ração comercial, pois a propriedade irá adquirir os ingredientes, para fazer o balanceamento da dieta de acordo com cada categoria animal e com cada lote da propriedade.

\subsection{Quinto ponto fraco: presença de lama nas áreas de descanso}

No presente estudo, observou-se a presença de lama nas áreas de descanso, o que foi considerado um ponto fraco. De acordo com Mauchle et al. (2008), as afecções podais em bovinos leiteiros têm origem multifatorial. Souza (2002) relatou que há aumento da ocorrência de problemas de 
casco no período das chuvas devido a formação de grande quantidade de lama, principalmente nas áreas próximas aos cochos de alimentação.

$\mathrm{O}$ alto teor de umidade e a presença de lama também contribuem para o aumento das infecções mamárias. Risseti et al. (2013) relataram que a mastite figura dentre as principais manifestações clínicas em vacas e novilhas, devido a veiculação das bactérias por moscas ou por via ascendente para o canal do teto, quando os animais se deitam em áreas com a presença de lama, aumentando a incidência em períodos do ano com maior temperatura e pluviosidade.

\subsection{Ferramenta proposta para solucionar o quinto ponto fraco: diagrama de Ishikawa}

Para solucionar o ponto fraco 'Presença de lama nas áreas de descanso' foi proposto o Diagrama de Ishikawa (Ishikawa, 1985), como ferramenta de gestão, devido a quantidade de variáveis envolvidas para a resolução do problema. O Diagrama de Ishikawa, também conhecido como Diagrama de Causa e Efeito ou Diagrama Espinha-de-peixe, é uma ferramenta gráfica utilizada para o gerenciamento e controle da qualidade em processos diversos. Para Ishikawa (1985), a composição do diagrama considera que os problemas podem ser classificados em sete tipos diferentes de causas. São os 7Ms: Machine (Máquina), Method (Método), Mother Nature (Meio Ambiente), Man Power (Mão de Obra), Material (Matéria-prima), Management (Gestão) e Measurement (Medida)).
Em alguns casos pode-se utilizar outro "M", o das Finanças (Money).

Para construir o diagrama, primeiramente, define-se o problema, ou efeito, a ser analisado. Em seguida, pode ser feito um breve Brainstorming para evidenciar a maior quantidade de causas que contribuem para o surgimento do problema; para tanto, basta perguntar o por quê de aquele problema estar acontecendo. O diagrama de Ishikawa pode ser visualizado na Figura 1.

Para que isso ocorra é necessário corrigir problemas secundários, que levam à presença de lama na área de descanso, tal como o manejo. Porém, cada problema secundário necessita ter as suas próprias causas resolvidas. Por exemplo, para que o problema secundário Manejo seja solucionado é preciso trabalhar dois itens fundamentais: a mudança constante de local da sombra (caso seja de sombrite), além do abaulamento de corredores. Caso alguns desses itens secundários não sejam minimizados, não se conseguirá resolver o ponto fraco principal. Quanto aos problemas secundários pluviosidade e terreno, eles não podem ser corrigidos após a implantação do sistema de produção. A avaliação desses dois itens deve ser feita de forma preventiva, ao se realizar a elaboração do projeto (planejamento) da atividade agropecuária. Este exemplo demonstra o quanto é complexa a resolução do problema primário; porém factível, como proposto na Figura 1.

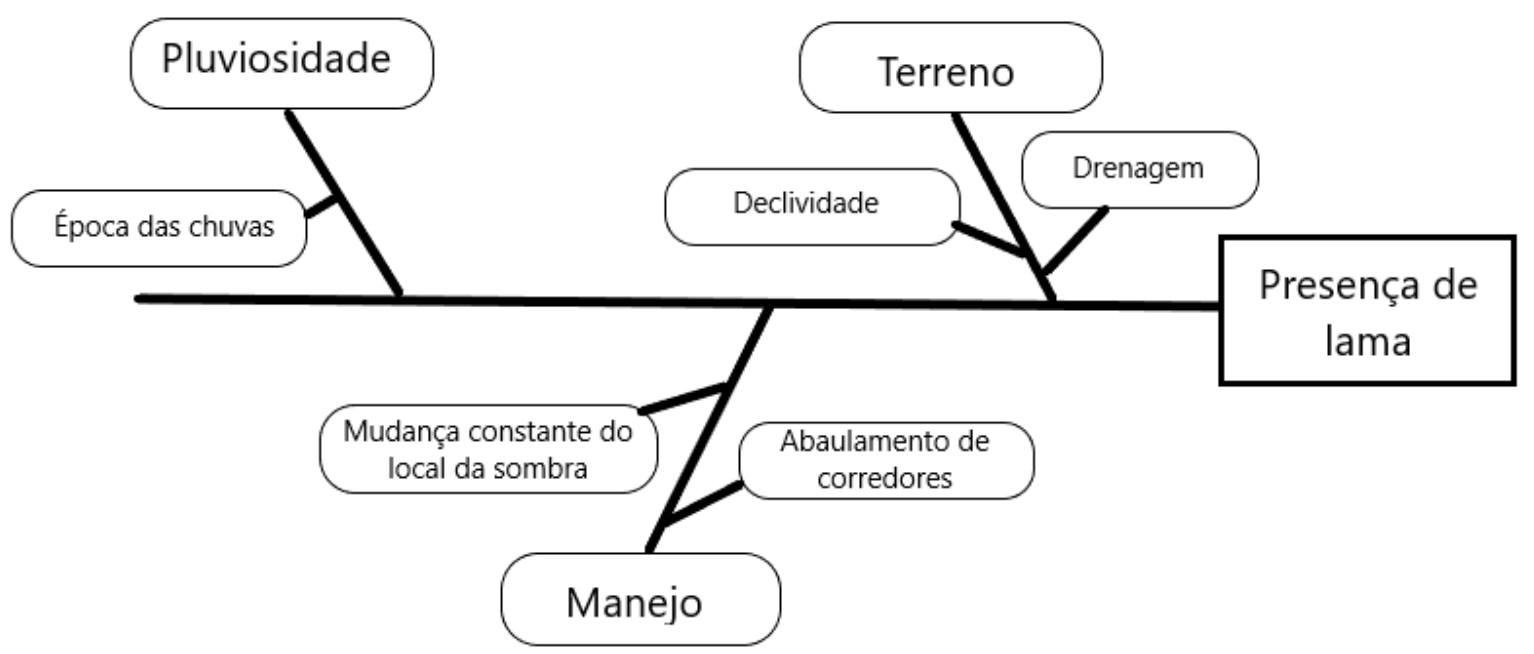

Figura 1 Diagrama de Ishikawa como proposta para resolução do ponto fraco presença de lama na área de descanso 


\subsection{Outros pontos fracos}

No Figura 2 podem ser observados outros pontos fracos encontrados nos sistemas de produção estudados, bem como a recomendação de ferramentas de gestão a serem utilizadas na expectativa de corrigi-los, ou mesmo minimizálos, para que esses não se tornem ameaças aobom desempenho produtivo e à rentabilidade das propriedades.

Figura 2 Pontos fracos identificados durante o diagnóstico do sistema de produção estudado, de agosto a dezembro de 2014, e recomendação da ferramenta de gestão a serutilizada

\begin{tabular}{|c|c|}
\hline Pontos fracos & Ferramenta \\
\hline Falta de controle de nas enfermidades brucelose e tuberculose bovinas & PDCA \\
\hline Ausência de pastejo rotacionado & $5 \mathrm{~W} 2 \mathrm{H}$ \\
\hline Falta de volumoso para a época seca & $5 \mathrm{~W} 2 \mathrm{H}$ \\
\hline Excesso de proteína fornecida aos animais & $5 \mathrm{~W} 2 \mathrm{H}$ \\
\hline Presença de lama nas áreas de descanso & Diagrama de Ishikawa \\
\hline Não faz vacinação de clostridiose, paratifo e leptospirose & $5 \mathrm{~W} 2 \mathrm{H}$ \\
\hline Baixa frequência de calagem e adubação & $5 \mathrm{~W} 2 \mathrm{H}$ \\
\hline Baixa proporção de vacas em lactação & PDCA \\
\hline Pastagem de baixa qualidade & Diagrama de Ishikawa \\
\hline Uso incorreto de sêmen sexado & $5 \mathrm{~W} 2 \mathrm{H}$ \\
\hline Pastejo diferido & $5 \mathrm{~W} 2 \mathrm{H}$ \\
\hline Troca local de casinha somente quando forma barro & $5 \mathrm{~W} 2 \mathrm{H}$ \\
\hline Compra de ração comercial & $5 \mathrm{~W} 2 \mathrm{H}$ \\
\hline Não vermifuga novilhas e vacas pré-parto & $5 \mathrm{~W} 2 \mathrm{H}$ \\
\hline Não faz análises de água & $5 \mathrm{~W} 2 \mathrm{H}$ \\
\hline Critérios errôneos de agrupamentos do rebanho & Diagrama de Ishikawa \\
\hline Não tem acasalamento genético & PDCA \\
\hline Baixa lotação & PDCA \\
\hline Joga fora o leite de transição & $5 \mathrm{~W} 2 \mathrm{H}$ \\
\hline Não possuirecomendação veterinária para tratamento da mastite & $5 \mathrm{~W} 2 \mathrm{H}$ \\
\hline Local inadequado para casinha das bezerras & $5 \mathrm{~W} 2 \mathrm{H}$ \\
\hline Critério errôneo para controle de infestação de ectoparasitas & PDCA \\
\hline Descorna realizada tardiamente & $5 \mathrm{~W} 2 \mathrm{H}$ \\
\hline Não utiliza dieta de transição & $5 \mathrm{~W} 2 \mathrm{H}$ \\
\hline Presença de plantas invasoras nos piquetes & PDCA \\
\hline Posição inadequada de bezerreiros & $5 \mathrm{~W} 2 \mathrm{H}$ \\
\hline Falta de manutenção de cercas & $5 \mathrm{~W} 2 \mathrm{H}$ \\
\hline Não faz vacinação pré parto & $5 \mathrm{~W} 2 \mathrm{H}$ \\
\hline Árvores com copa densa & $5 \mathrm{~W} 2 \mathrm{H}$ \\
\hline Fornecimento incorreto de concentrado para bezerras & $5 \mathrm{~W} 2 \mathrm{H}$ \\
\hline Baixa Produção vaca/dia & PDCA \\
\hline Identificação tardia de bezerras & $5 \mathrm{~W} 2 \mathrm{H}$ \\
\hline Não possuiconstância na pesagem das bezerras & $5 \mathrm{~W} 2 \mathrm{H}$ \\
\hline Não realiza biocarrapatograma & $5 \mathrm{~W} 2 \mathrm{H}$ \\
\hline Intervalo do controle leiteiro & $5 \mathrm{~W} 2 \mathrm{H}$ \\
\hline Não realiza OPG & $5 \mathrm{~W} 2 \mathrm{H}$ \\
\hline Cochos de sal descobertos & $5 \mathrm{~W} 2 \mathrm{H}$ \\
\hline Não possuibanco de colostro & $5 \mathrm{~W} 2 \mathrm{H}$ \\
\hline Não avalia a qualidade do colostro & $5 \mathrm{~W} 2 \mathrm{H}$ \\
\hline Intervalo entre ordenhas & $5 \mathrm{~W} 2 \mathrm{H}$ \\
\hline Ausência de irrigação de pastagens & Diagrama de Ishikawa \\
\hline Distância até o laticínio & PDCA \\
\hline Armazenamento do leite por $48 \mathrm{~h}$ & $5 \mathrm{~W} 2 \mathrm{H}$ \\
\hline
\end{tabular}




\subsection{Pontos Fortes}

Nos diagnósticos realizados foram observados pontos fortes nas propriedades analisadas. A sua identificação possibilita, aos técnicos, proprietários e pesquisadores um trabalho de maximização onde, será possível aumentar a produtividade e, consequentemente, a rentabilidade das propriedades. No que diz respeito à alimentação, os pontos fortes foram: uso de concentrado, alimentação é fornecida no cocho, uso de sal mineral específico para leite e uso de sal mineral à vontade, fornecido no cocho.

Quanto ao manejo de bezerras: Os animais recém-nascidos são bem cuidados, realiza-se a cura do umbigo com iodo por três dias seguidos; realiza higienização dos tetos para a ordenha do colostro; bezerras mamam o colostro até seis horas após o parto; bezerras bebem de 3 a 4 litros de colostro na mamadeira; quantidade correta de leite fornecida às bezerras até o desaleitamento; horário de amamentação das bezerras sempre constante e fornece o leite morno.

Em relação à reprodução, verificou-se: uso de inseminação artificial; uso de sêmen sexado; faz observação de cio duas vezes ao dia; as vacas que estão para parir são observadas várias vezes ao dia; e não utiliza touros para repasse.

Ordenha sem bezerro ao pé, adequada manutenção do equipamento de ordenha, troca das teteiras a cada seis meses, horário de ordenha sempre constante, higienização dos utensílios usados na ordenha com água e sabão apropriado, curral coberto e armazenamento do leite em tanques de expansão, bem como boa qualidade do leite com CBT menor que $10.000 \mathrm{ufc} / \mathrm{mL}$ e CCS menor que $230.000 \mathrm{cel} / \mathrm{mL}$, foram os pontos fortes observados em relação ao manejo de ordenha e qualidade do leite. Vale salientar que, no que diz a qualidade do leite, a Instrução Normativa $\mathrm{n}^{\mathrm{o}} 62$ do Ministério da agricultura Pecuária e Abastecimento preconiza requisitos mínimos de CBT de 100 mil ufc/mL e CCS menor que $400.000 \mathrm{cel} / \mathrm{mL}$.

Quanto ao manejo de vacas percebeu-se: pretensão de aumentar média de produção de leite por animal/dia (14 para $18 \mathrm{~kg}$ por dia), manejo adequado de vacas secas, identificação dos animais feita com brincos, secagem da vaca com bom escore de condição corporal (ECC) e de acordo com o estágio de gestação, vacas entram no piquete maternidade, que são sombreados, com 15 dias antes do parto, poucas incidências de distocias, retenção de placenta e abortamentos, assim como, baixa infestação de carrapatos, mosca de chifres e bernes. No que tange às instalações, os principais pontos fortes observados foram: presença de pedilúvio, nascente de água, energia elétrica, bebedouros nos principais locais da propriedade com presença de bóias, tais como instalações e piquetes, dimensionamento correto dos cochos e bebedouros, boa condução de práticas agrícolas (análise de solo, calagem e adubação, rotação de culturas e plantio direto).

Quando se trata da questão gestão de pessoas observou-se: não utilizar trabalhador temporário, que é considerado um ponto forte, além de sempre haver capacitação para os funcionários e técnicos e há interesse em crescimento profissional.

\section{Conclusão}

Conclui-se, portanto, que há possibilidades de adaptar as ferramentas de gestão, tão utilizadas em outras atividades, tais como indústria automobilística, indústria têxtil, gerenciamento de qualidade entre outras, na pecuária leiteira, visando à correção de pontos falhos e, consequentemente, o aumento da rentabilidade.

O uso das ferramentas de gestão se torna muito útil dentro da propriedade, atuando na identificação do problema, e os ordenando conforme sua gravidade, urgência e tendência a piorar.

O formulário empregado foi uma ferramenta fundamental para a realização da pesquisa e mostrou-se apto a ser utilizado em novos estudos de diagnóstico de propriedades de pecuária de leite.

O conhecimento da aplicação de ferramentas de gestão em fazendas leiteiras, gerado por esta pesquisa, abrirá caminho para construção de um plano estratégico de gestão, a partir de metodologias de diagnóstico organizacional e sistema de administração, e para consolidar a tomada de decisões, além de contribuir para a capacitação de recursos humanos em administração de empreendimentos rurais.

\section{Refe rências}

Abdalla, A. L.; Louvandini, H.; Bueno, I C S.; Vitti, D. M. S. S.; Meirelles, C. F; Gennari, S. M. Constraints to milk production in grazing 
dairy cows in Brazil and management strategies for improving their productivity. Preventive Veterinary Medicine, v.38, n. 1, p.217-230, 1999. https://doi.org/10.1016/S0167-5877(98)00127-5

Abreu, D. C.; Lana, R. P.; Oliveira, A. S.; Paula, R. M.; Rodrigues, J. P.; Ghedini, C.; Andrade, F. L.; Fonseca, M. A. Ureia de liberação lenta em dietas à base de cana-de-açúcar para vacas mestiças Holandês-Zebu. Revista Agrarian, v.8, n.30, p.399-404, 2015. https://doi.org/10.30612/agrarian.v8i30.2671

Aguiar, S. Integração das ferramentas da qualidade ao PDCA e ao programa seis sigma. 2 ed., Nova Lima: INDG, 2006. 234p.

Alves, I., Rezende, S. O., Oliveira Neto, O. J. de; Drees, C., Santana, R. Aplicação do modelo e análise SWOT no diagnóstico estratégico de uma propriedade rural especializada em recria e engorda de bovinos de corte. Revista Administra-Ação. v.1, n.1, p.22-39, 2007. http://periodicos.unievangelica.edu.br/index.php/administ racao/article/view/353

Andrade, S. P.; Boff, C. D. S. Ferramentas de planejamento para tomada de decisão aplicadas a um microempreendedor individual (MEI). Revista Contabilidade, Ciência da Gestão e Finanças, v. 2, n.1, p. 57-85, 2014. http://ojs.fsg.br/index.php/rccgf/article/view/1252

Antunes, L. M.; Essels, A. Agroqualidade: qualidade total na agropecuária. Porto Alegre: Guaíba Agropecuária, 1999. 113p.

Batalha, M. O.; Buainain, A. M.; Souza Filho, H. M. Tecnologia de gestão e agricultura familiar. São Carlos: UFSCar, 2005. 19p.

Bernués, A.; Manrique, E.; Maza, M. T. Economic evaluation of bovine brucellosis and tuberculosis eradication programmes in a mountain area of Spain. Pre ventive Veterinary Medicine, v.30, n.2, p.137-149, 1997. https://www.ncbi.nlm.nih.gov/pubmed/9234417

Bonamigo, A.; Melz, M. J.; Sehnem, S.; Winch, C. A Manejo dos dejetos de suínos através do sistema de compostagem. Revista em Agronegócios e Meio Ambiente, v.7, n.1, p. 659-681, 2014. http://periodicos.unicesumar.edu.br/index.php/rama/articl e/view/2946

Borges, A. L. C. C., Silva, R. R.; Gonçalves, L. C.; Molina, P. C.; Souza, A. S. O uso da canade-açúcar com ureia na alimentação de bovinos. Cadernos Técnicos de Veterinária e
Zootecnia, v.80, n.1, p.39-55, 2016. https://vet.ufmg.br/ARQUIVOS/FCK/file/editora/caderno \%20tecnico\%2080\%20ureia\%20dietas\%20ruminantes.pd $\underline{\mathrm{f}}$

BRASIL, Ministério da Agricultura, Pecuária e Abastecimento. Secretaria Nacional de Defesa Agropecuária. Programa Nacional de Controle e Erradicação da Brucelose e da Tuberculose Animal (PNCEBT). Instrução Normativa - IN $n^{\circ} 10$, de $3 / 3 / 2017$.

Cavalcante, M. A. B.; Pereira, O. G.; Valadares Filho, S. de C.; Ribeiro, K. G.; Chizzotti, F. H. M.; Pereira, D. H Níveis de proteína bruta em dietas para bovinos de corte: consumo e digestibilidades total e parcial dos nutrientes. Revista Brasileira de Zootecnia, v.34, n.6, p.2200-2208, 2005. http://dx.doi.org/10.1590/S1516$\underline{35982005000700006}$

Cheng, L. C.; Meli Filho, L. D. R. QFD: desdobramento da função qualidade na gestão de desenvolvimento de produtos. 2 . ed. Belo Horizonte: Edgard Blucher, 2007. 568 p.

Coletti, J.; Bonduelle, G. M.; Iwakiri, S. Avaliação de defeitos no processo de fabricação de lamelas para pisos de madeira engenheirados com uso de ferramentas de controle de qualidade. Revis ta Acta Amazônica, v.40, n. 1, p.135-140, 2010. http://dx.doi.org/10.1590/S0044$\underline{59672010000100017}$

Crepaldi, S. A. Contabilidade ge rencial: São Paulo. Atlas, 1998. 201p.

Duarte. L.; Tamashiro, H. R. S.; Merlo, E. M. Marcas próprias: uma análise da interface entre fornecedores de produtos lácteos e varejistas. In: XVI Simpósio de administração da produção, logística e operações internacionais, 16, 2013, São Paulo. Anais... São Paulo, SP, SIMPOI, 2013, p. 245-247.

Farias, A. P. S.; Fontana, M. E.; Morais, D. C. Modelo de sistema de informação e decisãopara intervenções de reabilitação em redes de distribuição de água. Revista B rasileira de Recursos Hídricos, v.18, n.2, p.4-16, 2013. https://abrh.s3.sa-east-

1.amazonaws.com/Sumarios/98/191a76d0b50e32b9cd50f b0cafb4c953 2b523b6d0cb31f4f553e5ff725c212a9.pdf

Houtert, M. F. J. V; Sykes, A. R. Enhancing the profitability of pasture-based dairy production in the humid tropics through improved nutrition. Preventive Veterinary Medicine, v.38, n.2-3, 
p. 147-157,

https://www.ncbi.nlm.nih.gov/pubmed/10081794

1999.

Ishikawa, K. Controle de qualidade total: à maneira japonesa. 3 ed.. Rio de Janeiro: Campus, 1985. 277p.

Lisbôa, M. G. P; Godoy, L. P. Aplicação do método $5 \mathrm{~W} 2 \mathrm{H}$ no processo produtivo do produto: a joia. Iberoamerican Journal of Industrial Engineering, v.4, n.1, p.32-47, 2012.

http://incubadora.periodicos.ufsc.br/index.php/IJIE/article $\underline{\text { /view/1585 }}$

Lopes, M. A.; Lima, A. L. R.; Carvalho, F. D. M.; Reis, R. P.; Santos, I. C.; Saraiva, F. H. Controle gerencial e estudo da rentabilidade de sistemas de produção de leite na região de Lavras (MG). Ciência e Agrotecnologia, v.28, n.4, p.883892, 2004. http://dx.doi.org/10.1590/S1413$\underline{70542004000400022}$

Lopes, M. A.; Reis, E. M. B; Ferrazza, R. A. Formulário de diagnóstico da propriedade leiteira. Lavras: UFLA, 2016. 19p. (Boletim Técnico)

Macedo, M. C. M. Integração lavoura e pecuária: o estado da arte e inovações tecnológicas. Revista B rasileira de Zootecnia, v.38, n.1, p.133-146,

2009. http://www.scielo.br/pdf/rbz/v38nspe/v38nspea15.pdf

Mauchle, U.; De Carvalho, A. Ú.; Alzamora Filho, F.; Ferreira, P. M.; Facury Filho, E. J.; Cavalcante, M. P. Efeito da sazonalidade sobre a ocorrência de lesões podais em vacas de raças leiteiras. Revista Brasileira de Saúde e Produção Animal, v.9, n.1, p. 109-116, 2008. http://revistas.ufba.br/index.php/rbspa/article/viewArticle/ $\underline{857}$

Meireles, M. Ferramentas administrativas para identificar, observar e analisar proble mas : organizações com foco no cliente. 2 ed., São Paulo; Arte \& Ciência, 2001. 122p.

Mertens, D.R. Regulation of forage intake. In: Forage quality, evaluation and utilization. Fahey J. R (ed.) American Society of Agronomy: Forage quality, evalution and utilization, Madison, 1994, Chapter 4, p. 450493.

Michel, A. L.; Muller, B.; Helden, P. D. Mycobacterium bovis at the animal-human interface: a problem, or not. Veterinary Microbiology, v.140, n.3, p.371-381, 2010. https://doi.org/10.1016/j.vetmic.2009.08.029
Miranda, K. L.; Alves, C. M.; Minharro, S.; Lobo, J. R.; Müller, E. E.; Gonçalves, V. S. P.; Lage, A. P. Quem ganha com a certificação de propriedades livres ou monitoradas pelo PNCEBT? Re vista do Leite Integral, v.3, n. 1, p.44-55, 2008.

Mocci, D.; Renesto, D. M.; Rodrigues, A. R. A.; Silva, R. S.; Machado, J. G. Prevalência da brucelose e tuberculose bovina em propriedades da região de São José do Rio Preto-SP. ARS Veterinaria, v.30, n.2, 100-103, 2014. http://dx.doi.org/10.15361/2175-0106.2014v30n2p 100$\underline{103}$

Moreira, H. A. Cana-de-açúcar na alimentação de bovinos. Informe Agropecuário, v.9, n.108, p. 14-16,1983.

Nascimento, R. S.; Carvalho, N. L. Integração lavoura-pecuária. Revista Monografias Ambientais, v.4, n.4, p.828-847, 2011. http://dx.doi.org/10.5902/223613083553

Novo, A. L. M.; Schiffler, E. A. Princípios básicos para produção econômica de leite. São Carlos: Embrapa Pecuária Sudeste, 2006. $23 \mathrm{p}$.

Oliveira, S. T. Ferramentas para o aprimoramento da qualidade. 2. ed. Rio de Janeiro: Guanabara Koogan, 1996. 119p.

Oliveira, V. S.; Morais, J. A.; Fagundes, J. L.; Lima, I. G.; Santana, J. C.; Santos, C. B. Efeito da irrigação na produção e qualidade de pastagens durante o período da seca. Revista Científica Eletrônica de Medicina Veterinária, v.26, n.1, 175-192, 2016. https://www.bvs-vet.org.br/vetindex/periodicos/revistacientifica-eletronica-de-medicina-veterina/26(2016)/efeito-da-irrigacao-na-producao-e-qualidade-depastagens-durante-o-per/

Parré, J. L.; Bankuti, S. M. C.; Zanmaria, N. A. Perfil socioeconômico de produtores de leite da região sudoeste do Paraná:um estudo a partir de diferentes níveis de produtividade. Revista de Economia e Agronegócio v.9, n.1, 275-300, 2011. http://dx.doi.org/10.25070/rea.v9i2.187

Pereira, M. N.; Resende, J. C.; Pereira, R. A. N.; Silva, H. C. Indicadores de desempenho de fazendas leiteiras de Minas Gerais. Arquivos Brasileiros de Medicina Veterinária e Zootecnia, v.68, n.4, p.1033-1042, 2016. http://dx.doi.org/10.1590/1678-4162-8218

Peron, A. J.; Evangelista, A. R. Degradação de pastagens em regiões de cerrado. Ciência e 
Agrotecnologia, v.28, n.3, p.655-661, 2004. http://dx.doi.org/10.1590/S1413-70542004000300023

Poester, F. P.; Samartino, L. E.; Santos, R. L. Pathogenesis and pathobiology of brucellosis in livestock. Scientific and Technical Review of the Office Inte rnational des Epizooties, v.32, n.1, p.105- 115, 2013. http://web.oie.int/boutique/extrait/11poester105115.pdf

Polacinski, E.; Silva, V. B.; Tauchen, J. O. E. L.; Pires, M. R. Implantação dos 5Ss e proposição de um SGQ para uma indústria de erva-mate. In: Congresso internacional de administração. Gestão estratégica: Empreendedorismo e Sustentabilidade. 1. 2012. Ponta Grossa. Anais... Ponta Grossa: UFPG, 2012, p.64-66.

Reis, R. A.; Rosa, B. Suplementação volumosa: conservação do excedente das pastagens. In: Simpósio sobre manejo da pastagem, 18, 2001, Piracicaba Anais... Piracicaba: FEALQ, 2001. p. 193-232.

Ribeiro, V. C. G.; Medeiros, R. R.; Almeida, T. M.; Ferrari, M. C.; Das Graças Souza, M. Análise da viabilidade econômica do pastejo rotacionado irrigado na pecuária leiteira. In: $5^{\mathrm{a}}$ Jornada científica e tecnológica da FATEC, 5., 2016, Botucatu. Anais... Botucatu: UNESP, 2016, p.74-79.

Risseti, R. M.; Lara, G. H. B.; Franco, M. M. J.; De Moraes, A. B. C.; De Oliveira Zamprogna, T.; Bolanos, C. A. D.; Ribeiro, M. G. Perfil de sensibilidade microbiana 'in vitro' em linhagens de trueperella (Arcanobacterium) pyogenes isoladas de mastite bovina. Veterinaria e Zootecnia, v.20, n.2, supl.1, p.96-104. 2013. http://www.fmvz.unesp.br/rvz/index.php/rvz/article/view/ $\underline{707 / 456}$

Roberti, F.; Santana, S. Melhoramentos e padronização de processos em restaurante. Reunião Anual de Ciências, v.3, n.1, p.73-82, 2013.

http://www.computacao.unitri.edu.br/erac/index.php/e$\underline{\text { rac/article/view/36 }}$

Santana; M. G.; Araújo, M. B. V. Aplicação do PDCA na resolução do acréscimo de água no leite cru recebido em um laticínio no estado do Pará. Cadernos de Pós-Graduação da FAZU, v. 2, n.2, p.33-39, 2011. http://www.fazu.br/ojs/index.php/posfazu/article/view/41 $\underline{1}$

Santiago, A. M. F., Campos, J.M.; Oliveira, A.S.; Valadares Filho, S. C.; Santos, S.A.; Souza,
S.M.; Santiago, I. F. Urea in sugarcanebased diets for dairy cows. Revista B rasileira de Zootecnia, v.42, n.6, p.456-462, 2013. http://dx.doi.org/10.1590/S1516-35982013000600010

Santos, R. L.; Martins, T. M.; Borges, Á. M.; Paixão, T. A. Economic losses due to bovine brucellosis in Brazil. Pesquisa Veterinária Brasileira, v.33, n.6, p.759-764, 2013. http://dx.doi.org/10.1590/S0100-736X2013000600012

Silva, M. F.; Pereira, J. C.; Gomes, S. T.; Nascif, C.; Gomes, A. P. Avaliação dos indicadores zootécnicos e econômicos em sistemas de produção de leite. Revista de Política Agrícola, v.24, n.1, p.62-73, 2015. https://seer.sede.embrapa.br/index.php/RPA/article/view/ $\underline{967}$

Sousa, E. P. M.; Silva, E.T.; Marques, A. Aplicação das ferramentas da qualidade emuma gráfica norteriograndense. In: VI Simpósio de Engenharia de Produção da Região Nordeste, 2, 2011, Campina Grande, Anais... Campina Grande: UFPB, 2011, p. 46-47.

Souza, A. C.; Andrade, L. S. S.; Sousa, D. L. S Orientações para o descarte responsável de medicamentos de uso veterinário. In: XIII Jornada de ensino, pesquisa e extensão, 2, 2013, Recife. Anais... Recife: UFRPE, 2013, p.161163.

Souza, R. C. Perfil epide miológico e clínico das afecções podais em vacas nas bacias leiteiras de BeloHorizonte e Pedro Leopoldo. 2002. 57 f. Dissertação (Mestrado) - Universidade Federal de Minas Gerais, Belo Horizonte.

Souza, R. C.; Reis, R. B.; Lopes, F.; Mourthe, M. H. F.; Lana, A. M. Q.; Barbosa, F. A.; Sousa, B. $M$ Efeito da adição de teores crescentes de ureia na cana-de-açúcar em dietas de vacas em lactação sobre a produção e composição do leite e viabilidade econômica. Arquivo B rasileiro de Medicina Veterinária e Zootecnia, v.67, n.2, p.564-572, 2015. http://dx.doi.org/10.1590/1678$\underline{7799}$

Valadares Filho, S. C.; Valadares, R. F. D. Recentes avanços em proteína na nutrição de vacas leiteiras. In: Simpósio internacional de bovinocultura de leite, 2, 2001, Lavras. Anais... Lavras: UFLA, 2001. p.228-243.

Valle, C. B.; Euclides, V. P. B.; Macedo. M. C. M. Características das plantas forrageiras do gênero Brachiaria. In: Simpósio sobre manejo 
Uso de ferramentas de gestão na atividade leiteira: um estudo multicasos em Uberlândia, MG

Lopes et al.

da pastagem, 17., 2000, Piracicaba. Anais... v.95, n.3, p.4176-4183, 2012. Piracicaba: FEALQ, 2000. p. 65-108.

https://doi.org/10.3168/jds.2011-5219

Wolf, C. A. Dairy farmer use of price risk management tools. Journal of Dairy Science, 